\title{
Using In-Situ TEM to Characterize the Microstructure Evolution of Metallic Systems under External Solicitation
}

\author{
D. Kaoumi ${ }^{1}$, T. Gautier ${ }^{1}$, J. Adamson ${ }^{1}$, M. Kirk ${ }^{2}$ \\ 1. University of South Carolina, 300 Main St., SC, 29208, USA, djamelkaoumi@gmail.com \\ 2. Argonne National Laboratory, Bldg 212, IL, 60439 USA, kirk@anl.gov
}

Studying materials under external stimulus such as irradiation and/or mechanical stress can be difficult because of the lack of kinetics information, since usually samples are examined ex situ (e.g. after irradiation or after mechanical testing) so that only discrete snapshots of the process are available. Given the dynamic nature of the phenomena, direct in situ observation is often necessary to better understand the mechanisms, kinetics and driving forces of the processes involved. For this matter, using in situ Transmission Electron Microscopy (TEM) can be of great help[1]. Indeed, the spatial resolution of the TEM makes it an invaluable tool in which one can continuously track the real-time response of the microstructure to external stimuli, which can help discover and quantify the fundamental rate-limiting microscopic processes and mechanisms governing the macroscopic properties. In this presentation, two examples will be given which show how the technique can be used for nuclear engineering applications. (i) In-situ straining experiments in the TEM is applied to investigate deformation mechanisms in Ni-based alloys (Inconel 617 and Haynes 230) which are candidate materials for the heat exchanger in the GEN-IV Very High Temperature nuclear Reactor. In addition to showing dislocation dynamics under tensile strain, it also allows to follow crack propagation as it proceeds in the material. (ii) In-situ Ion-irradiation in the TEM has proven a very good tool for studying the basic mechanisms of radiation damage formation and evolution as a function of dose, dose rate, temperature and ion type.

In-situ straining in a TEM: Foils of Ni-based Inconel 617 were deformed in situ in the TEM at $298 \mathrm{~K}$ and $573 \mathrm{~K}$. The dynamic observations of the deformation processes are described especially in terms of dislocation motion and interaction, (e.g. dislocation (double) cross-slip and annihilation). The alloy presents dislocation slip bands in its as-received state (un-aged sheet), and several additional slip bands develop during the straining experiments. The movement of the dislocation through the slip bands, at the interfaces, and about the precipitates is observed in-situ as illustrated in Figure 1. The arrangement of moving dislocations, especially the propagation of pile-ups of bowed dislocations, is observed in detail. The dislocations moving in the slip bands are not the preexisting dislocations but originate from grain boundaries and other stress concentrators. Also, dynamic observations of crack initiation and growth mechanisms are obtained in the same experiments. Intragranular crack initiation and propagation is also observed in the same samples. The crack propagation in the un-aged alloy appears to occur mostly in a zigzag manner. Microcracks form at stress concentration sites in front of the major crack and then the major crack advances by microcrack linkage. Similar modes of fracture are observed at 300K, 573K[2]. The main observations will be reported and the possible mechanisms discussed.

In-situ ion irradiation in a TEM: Ion-irradiation is a useful way to reach high level of radiation damage doses in a reasonable amount of time with a damage structure that can be similar to that achieved under neutron irradiation without the complications of radioactivity. When combined with Transmission Electron Microscopy it becomes a powerful tool to gain kinetics information on 
irradiation induced phenomena because the microstructure evolution of the irradiated material can be followed in-situ as the damage proceeds. Using this technique the microstructure evolution under irradiation was studied in two model F/M steels (9Cr and 12Cr ) irradiated with $1 \mathrm{MeV} \mathrm{Kr}$ ions insitu at temperatures between $20 \mathrm{~K}$ and $573 \mathrm{~K}$ to doses as high as $10 \mathrm{dpa}$. During the early stages of irradiation of the two F/M steels, defect clusters appear to be rather uniformly distributed within grains, and the saturation density is quickly reached between 1.0 and $2.0 \mathrm{dpa}$. However, at doses as low as $3 \mathrm{dpa}$, self-ordered alignments of defect clusters are found in some grains. The regularly ordered arrays of small loops with spacing about 30-50 nm are observed in both F/M steels along $<110>$ directions. Once the aligned structure is created, it is stable under further irradiation. The possible mechanisms for the "self-organization" of the clusters were investigated. These structures are thought to result from elastic interactions between defect clusters in the foil. The fact that such defect alignment is not observed at higher temperatures suggests that the relatively high density of defect clusters (at lower temperatures) and the resultant internal strains may be the main reason for the development of the aligned structure. The preferred crystallographic orientation of defect arrays may be driven by the minimization of elastic interaction energy between defect clusters [3]. The defect self-ordering process, its temperature dependence and the possible mechanisms are discussed.

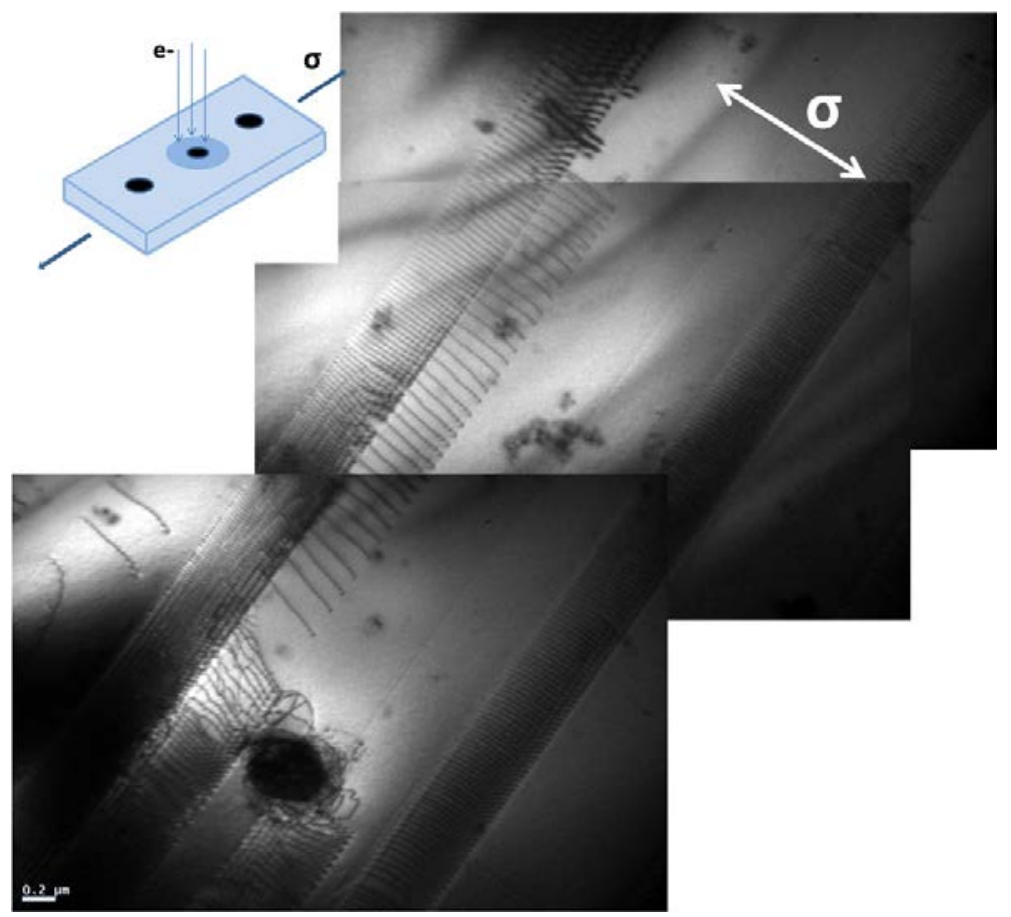

FIG. 1: TEM observations of Inconel 617 strained in-situ at 298K showing dislocation pile-ups and interaction with precipitate.

References:

[1] D. Kaoumi, A. Motta, R. Birtcher,Proceedings of the Workshop on the Use of In-Situ TEMIon Accelerator Techniques in the Study of Radiation Damage in Solids, The University of Salford, UK, 2009.

[2] D. Kaoumi T. Gautier, M. Kirk, 19th International Symposium on Plasticity, 2013, Nassau, Bahamas.

[3] D. Kaoumi, J. Adamson, Journal of Nuclear Materials, 448: p 233-238, 2014. 